Revue d'histoire de l'Amérique française

BEVUE D.HISTOIRE DE L'AMÉRIQUE FRANÇAISE

\title{
LAMONDE, Yvan et Didier POTHON, dir., La Capricieuse (1855) : poupe et proue. Les relationsFrance-Québec (1760-1914) (Québec, Les Presses de l’Université Laval, 2006), 379 p.
}

\section{Jacques-Guy Petit}

Volume 61, numéro 1, été 2007

URI : https://id.erudit.org/iderudit/016885ar

DOI : https://doi.org/10.7202/016885ar

Aller au sommaire du numéro

Éditeur(s)

Institut d'histoire de l'Amérique française

ISSN

0035-2357 (imprimé)

1492-1383 (numérique)

Découvrir la revue

Citer ce compte rendu

Petit, J.-G. (2007). Compte rendu de [LAMONDE, Yvan et Didier POTHON, dir., La Capricieuse (1855) : poupe et proue. Les relationsFrance-Québec (1760-1914) (Québec, Les Presses de l'Université Laval, 2006), 379 p.] Revue d'histoire de

l'Amérique française, 61(1), 119-123. https://doi.org/10.7202/016885ar d'utilisation que vous pouvez consulter en ligne.

https://apropos.erudit.org/fr/usagers/politique-dutilisation/ 
de retrouver, dans certains cas, des bibliographies incomplètes, alors que la majorité des articles n'en possède aucune. Si le manque d'uniformisation paraît déconcertant, cette lacune l'est tout autant, surtout que les outillages bibliographiques s'avèrent parfois très utiles pour le lecteur. Enfin, l'absence de biographies présentant les collaborateurs et leurs principaux champs de recherches est regrettable, surtout que, pour le lecteur québécois, près de la moitié des auteurs proviennent d'universités outre-Atlantique aussi diverses que l'Université de Marc Bloch de Strasbourg, l'Université de Paris 3, l'Université de Bari ou encore l'Université de Gênes.

Pour conclure, le livre Vers la construction d'une citoyenneté canadienne renferme des articles pertinents qui précisent le rôle des domaines scolaire et religieux dans la construction du multiculturalisme canadien et québécois. Ces derniers baignent toutefois dans une certaine ambiguiité en ce qui a trait à la nature du multiculturalisme, obligeant le lecteur à décortiquer difficilement une conceptualisation parfois laissée en suspens.

STÉPHANE SAVARD Département d'histoire et CÉLAT Université Laval

LAMONDE,Yvan et Didier POTHON, dir., La Capricieuse (1855): poupe et proue. Les relations France-Québec (1760-1914) (Québec, Les Presses de l'Université Laval, 2006), 379 p.

Tout semblait avoir été dit sur le voyage de La Capricieuse, ce navire de guerre français qui avait remonté le Saint-Laurent en 1855, événement considéré par la population canadienne-française du Bas-Canada comme le symbole du premier «retour» officiel de la France depuis la Conquête. Évidemment, pour la recherche historique, le dernier mot n'est jamais dit, le renouvellement des problématiques permettant de nouveaux éclairages. C'est le cas avec cet ouvrage qui présente les Actes du colloque tenu à Québec en 2005 pour le $150^{\mathrm{e}}$ anniversaire de la venue de la Corvette, dans le cadre de la Commission franco-québécoise sur les lieux de mémoire communs (Marcel Masse). Après le centenaire célébré à Terre-Neuve (J. M. Huille), c'est une belle occasion pour les historiens québécois et français de soumettre à la réflexion critique cette communauté mémorielle.

Les 17 communications se répartissent chronologiquement en trois ensembles d'inégale longueur: les relations entre la France et le Canada pendant l'ensemble de la période considérée; le voyage de La Capricieuse 
et son contexte; l'après 1855 . C'est ce que suggère la métaphore de la proue et de la poupe, mais une lecture thématique est aussi possible. Loin d'être une mission voulue par Napoléon III dans le cadre d'une nouvelle stratégie diplomatique concernant l'Amérique du Nord, la venue du vaisseau est une mission commerciale explicable par la conjoncture économique, par la diplomatie européenne et, plus prosaïquement, par l'ennui d'un capitaine de vaisseau qui supporte mal son séjour forcé à Terre-Neuve. Et la carrière de Belvèze, contrairement à ce qui a été souvent affirmé, n’a pas été cassée en raison de sa mission. Avec J. Portes, près de la moitié des textes reviennent sur ces démonstrations, mais on sait qu'un colloque échappe difficilement à la répétition.

La conjoncture est celle de l'abolition des Corn Laws (1846) et de l'abrogation des Actes de Navigation (1849-1852). Depuis la Conquête, le Canada est, commercialement aussi, "un pays perdu » et des ports comme La Rochelle, auparavant très actifs, se sont reconvertis dans la pêche morutière (D. Pothon). Les relations commerciales entre la France et le Canada, presque inexistantes avant 1850 , semblaient pouvoir se développer avec les mesures de libre-échange, mais ces deux pays resteront au $\mathrm{XIX}^{\mathrm{e}}$ siècle des partenaires marginaux (B. Marnot). Diplomatiquement, depuis la prise du pouvoir par l'anglophile Napoléon III, l'alliance franco-anglaise constitue un des piliers de la politique étrangère de la France, les deux pays se mettant d'accord pour bloquer l'expansion russe en Orient.

À l'été 1855 , la guerre de Crimée piétine et même si la France a ouvert des agences consulaires au Canada en 1850 (Québec et Sydney) et 1854 (Saint-Jean et Halifax), même si l'Empereur s'intéresse au sort des familles acadiennes, il n'est pas question d'indisposer l'Angleterre. L'Empereur n'a donc aucune visée sur le Canada-Uni, alors qu’en cette année 1855, les souverains s'invitent à Londres en avril puis à Paris en août, en pleine exposition universelle. Le voyage de La Capricieuse est bien provoqué par P.-H. Belvèze, commandant de la Station navale de Terre-Neuve qui souhaite aller au Canada pour concilier le plaisir d'une excursion et l'occasion de développer les échanges commerciaux. Avant d'obtenir l'accord des ministères concernés, il lui faudra beaucoup batailler, car si le ministre de la Marine est vite acquis, celui des Affaires étrangères est très réticent, par crainte d'importuner l'allié anglais. Les instructions qui parviennent à Belvèze lui donnent mission d'accomplir ce qu'il avait proposé, une mission purement commerciale, mais avec quelques précisions : préparer l'ouverture (déjà prévue) du Consulat de France à Montréal et négocier des droits de douane favorables. Il n'y aura donc aucun malen- 
tendu avec le gouvernement britannique (R. Pichette, J.-F. de Raymond, J. Portes).

Avant 1855, certes limités comparativement à ceux qui existaient avant 1760, les liens entre la France et le Canada sont loin d'être inexistants. Depuis 1815, la famille de libraires français Bossange a noué des relations d'amitié et de travail avec la famille Papineau, relations qui se poursuivront avec Hector Fabre. Par les librairies de Montréal, les échanges sont fréquents (livres, objets de piété et autres marchandises de France) et L.-J. Papineau sera aidé par les Bossange pendant son exil parisien de 18391845. Avec les insurrections de 1837-1838, la presse redécouvre momentanément son ancienne colonie mais, en dehors des journalistes républicains du National, elle manifeste peu d'intérêt pour ceux qu'elle qualifie d'«anarchistes» en reprenant les journaux britanniques. Les libéraux au pouvoir considèrent aussi que l'alliance anglaise est le meilleur rempart contre l'emprise des monarchies absolutistes en Europe. Papineau sera vite découragé de constater ce désintérêt qui s’aggravera en 1840 avec le tournant répressif du régime. Diplomatiquement, cet exil est donc un échec aggravé par ses difficultés financières et les désaccords avec les patriotes exilés aux États-Unis.

Dans ce contexte, les efforts de J.-G. Barthe, le greffier montréalais qui vient à Paris en juin 1853 pour "renouer le Bas-Canada avec la France», sont aussi voués à l'échec. Son ouvrage Le Canada reconquis par la France (janvier 1855) est ignoré ou critiqué, en particulier par Belvèze qui craint qu'il ne gêne sa mission. Depuis les voyages au Québec de $\mathrm{M}^{\mathrm{gr}}$ ForbinJanson en 1840 et celui d'Alexandre Vattemare (1839-1841) qui est à l'origine de la création de l'Institut canadien, les journaux et les livres français sont pourtant de plus en plus présents au Québec. Bien avant la venue de la Capricieuse, les ponts coupés diplomatiquement ont donc été en grande partie rétablis par l'imprimé et l'amitié (F. Labonté, F. Lejeune, Y. Lamonde, G. Gallicheau). Et, tout au long du siècle, en trois périodes principales, 1792-1840, 1840-1880 (avec l'action de $\mathrm{M}^{\text {gr }}$ Bourget), 18801914, prêtres et communautés religieuses de France irriguent le catholicisme québécois, favorisant ses orientations antirévolutionnaires (G. Laperrière).

De l'accueil triomphal de la Capricieuse à Québec, puis de la poursuite du voyage de Belvèze, sans son navire, à Montréal et dans le Haut-Canada, on retiendra l'importance, chez les Canadiens français, des symboles militaires, du drapeau tricolore, "signal d'alliance » avec «la vieille patrie» qui recoud la déchirure de la Conquête selon le poète charentais Jacques- 
Adolphe Marsais. Dans les discours, dans les journaux, le souvenir l'emporte sur l'histoire, tandis que pour la patrie ou la nation que l'on célèbre, les officiels sont partagés: «Albion notre foi, la France notre cœur» déclare E. P. Taché en reprenant Crémazie. Pour les observateurs, la visite de Belvèze à F.-X. Garneau et à Crémazie constitue un des sommets de la mission, mais les Rouges restent réticents devant des manifestations qui paraissent une démonstration de force en vue d'intimider les Américains. Les élites canadiennes-anglaises se montrent chaleureuses et célèbrent l'entente cordiale, ce qui n'empêchera pas le gouverneur général E. W. Head, dès octobre 1855, de reprendre les propos de Durham sur la supériorité de la "race» anglaise (P. Groulx, Y. Grisé).

La poupe ou le sillage de La Capricieuse ne fait l'objet que de peu d'études dans cet ouvrage, sans doute parce que cette visite restera pratiquement sans lendemains, du moins du côté français, en dehors de la création, tardive, du consulat commercial français (1859). Le leplaysien Rameau de Saint-Père, qui défend à Rome les intérêts des catholiques francophones, influence le mouvement de colonisation intérieure et il favorise le développement de la littérature francophone canadienne comme socle de l'idée nationale (P. Trépanier).

Quant à la reconstruction de l'idée nationale en France, elle devient une urgence après la défaite de 1870 et la perte de l'Alsace et de la Lorraine. Dans les manuels pour les écoles primaires publiques de la Troisième République, le genre du «tour de France» se développe afin d'inculquer aux enfants le code de la nation, par la reconquête symbolique du territoire. Le Canada y devient «l'autre province perdue» dont on conserve la mémoire pour réconforter la patrie meurtrie (P. Cabanel). André Siegfried évoque aussi la guerre de 1870 pour rappeler la sympathie que manifestèrent alors les Canadiens pour la France. Dans ses ouvrages de 1906 et 1914 sur le Canada et l'Amérique du Nord, ce protestant anglophile légitime la revendication nationaliste des Canadiens français qu'il ne croit possible que dans le cadre d'un compromis dont Laurier serait le meilleur artisan. Il ne situe la redécouverte du Canada par la France que vers la fin du XIx ${ }^{\mathrm{e}}$ siècle, depuis que des liens d'amitié et de commerce se développent (représentation québécoise à Paris en 1882, etc.), que se multiplient les visites d'officiels, avec notamment les trois voyages de Laurier à Paris entre 1896 et 1907. Gabriel Hanotaux et Raoul Dandurand sont les autres piliers de ce réseau de libéraux. Le Canada français intéresse la République comme le rappel du glorieux passé colonial, mais au début $\mathrm{du} \mathrm{xx}^{\mathrm{e}}$ siècle, le regard de la France change et le Canada qui commence à s'affranchir 
de la Grande-Bretagne devient un interlocuteur à part entière (G. Fabre).

Cet ouvrage éveille constamment l'intérêt et il renouvelle la question. Les remarques qu'il suscite restent donc mineures. Les relations entre la France et le Canada au $\mathrm{xIx}^{\mathrm{e}}$ siècle sont abordées sous de nombreux angles: commerciaux, diplomatiques, culturels, religieux. Mais pourquoi négliger les relations médicales et la forte influence de la médecine française au Québec (voir le poids des pasteuriens, les travaux de D. Goulet, etc.)? Tocqueville est plusieurs fois présenté comme celui qui, le premier depuis la Conquête, voyage au Canada et on lit même que Théodore Pavie le suit en 1832. Armand Yon avait pourtant bien relevé que Pavie était venu au Canada en 1829, deux ans avant Tocqueville.

Pour les échanges culturels, on pourrait rappeler que Chateaubriand est beaucoup lu au Bas-Canada autour de 1820 et que la Revue des deux mondes, dès son début en 1831, met le Canada à l'honneur en offrant au grand public cultivé de France un long «Essai historique, statistique et politique sur le Canada » (I: 376-417). Cette grande revue publiera d'autres articles sur le Canada avant la mission de La Capricieuse. Le Canada est donc constamment "redécouvert». Enfin, comme nous y invitent la solide conclusion de Yvan Lamonde et de Didier Pothon ainsi que le beau texte de Patrice Groulx, il resterait à s'interroger sur les effets de notre propension à commémorer. Si l'histoire et la mémoire entretiennent une féconde dialectique, ne fabrique-t-on pas parfois une sorte de mémoire artificielle? Quant au «lieu de mémoire commun», dans le cas précis de La Capricieuse, pendant et après l'événement, il est très ténu, mais il nous vaut ce beau livre sur l'ensemble des relations France-Québec au XIx ${ }^{\mathrm{e}}$ siècle.

JACQUES-GUY PETIT Université d'Angers CERHIO/CNRS et CERPECA

LEGENDRE, Camille, Le travailleur forestier québécois. Transformations technologiques, socioéconomiques et organisationnelles (Québec, Presses de l'Université du Québec, 2005), 397 p.

L’ouvrage de Camille Legendre entend présenter la foresterie québécoise et les diverses transformations qu'elle a connues depuis le début $\mathrm{du} \mathrm{xx}^{\mathrm{e}}$ siècle. Professeur de sociologie à l'Université de Montréal depuis de nombreuses années, l'auteur aborde les divers aspects du travail forestier au Québec à travers les cadres de la socio-économie et de la sociologie des 